1. Intrathecal injection of $\gamma$-amino- $\beta$-hydroxy-butylic acid and $\gamma$-aminobutyl1-histidine solution have been employed in 63 cases of epileptic patients who had uncontrolled attacks by ordinary medication of anticonvulsants. It has revealed that there were no more convulsive attacks on 28 cases, marked reduction of seizure on 18 cases, no significant change of their attacks on 11 cases, progressive state of the attacks on 2 cases in long range follow-up of the period over one and half years. And four cases of the epileptics had fatal convulsive attacks during these period.

2. $100 \mathrm{mg}$ of GABOB solution have been administered on the cerebral surface of the patients who underwent craniotomy and extirpation of their cerebral tumors. The administration was done just before closing the dura mater during surgery. Thus, there have been observed early postoperative convulsion in 13 cases out of 130 cases of GABOB administrated group and 6 cases out of 47 cases of control group. Convulsions were very mild in the 10 cases among 13 cases of the GABOB group, although severe in 4 cases among 6 cases of control group.

3. Recently, $\gamma$-amino- $\beta$-phenyl-butylic acid (P-GABA) and $\gamma$-amino- $\beta$ sulfonyl-butylic acid (S-GABA) have been noted to have an anticonvulsive action when they applied into cerebrospinal fluid directly. However, 20\% S-GABA and $1.5 \%$ P-GABA solution provoked spikes on the corticogram of the dog brain, where they applied directly on the cerebral surface experimentally. It is very interesting that those spikes were beautifully controlled or disappeared by means of the cortical application of $5 \% \mathrm{GABOB}$ solution in this experiment. Similar corticographic investigations were performed on the epileptogenic focus of the cerebral cortex of the patient who was explored to have his left frontal cortical scar by routine craniotomy. Application of 5\% S-GABA solution to the epileptogenic focus brought slight reduction of amplitude of background activities of his corticogram, but spikes were still presented. When 5\% GABOB solution was applied to the focus, the spikes were completely disappeared. There was, morover, no change of the other activity of his corticograms. Electrical stimulation of the focus provoked marked biphasic after-effect on his corticogram. Application of 5\% S-GABA solution brought practically no significant change to the after-discharge, although application of $5 \%$ GABOB solution showed marked inhibitory effect to it.

\title{
39. The EEG Change following Head-injury
}

\author{
Shuzo OKumura \\ Neurosurgical Service, National Hospital of Okayama
}

The EEG of 320 closed head injury cases was recorded in the standard fashion in their chronic stage between 1 to 12 months after the injury. The resting not activated record was evaluated according to the normal control record of 82 healthy 
persons.

Five percent of all head injury cases showed intermittent abnormality and 18 percent showed continuous abnormality. The incidence of abnormal was higher in groups those had skull fracture, positive gross neurological sign, history of convulsion, or experience of surgery for acute intracranial hematoma. However, no relation was observed between the incidence of EEG abnormality and the duration of post-traumatic unconsciousness. Intermittent abnormality was frequent $(23 \%)$ in the group younger than 9 year, and continuous abnormality was higher $(30 \%)$ in the group 10-19 year. Both type abnormalities showed low incidence in aged group. Then, it was suggested that post-traumatic EEG abnormality may depend on both severity of the injury and sensitivity of the brain under influence of the age.

At 39 cases sequential EEG record in several months interval was taken in the time between 1 to 12 months after the injury. About a half of the cases showed fluctuation of EEG findings, but no case showed marked change during the study. No relation was observed between fluctuating tendency of EEG and age or type of the injury. The fluctuation of EEG in those records was due to tentative fluctuation of the patient's condition and not maintenance change.

\title{
40. Averaging Analysis of Pelvic Afferent Potentials in Upper Brain Stem
}

\author{
Shinjiro Yamamoto, Tetsuro Yamamoto, Minoru Hayashi, \\ Shuso IzUMI, Kiyotoshi Yamano and Tomonori HanYU \\ Department of Neurosurgery, School of Medicine, University of Kanazawa
}

A study was made of the afferent projections of the pelvic nerve in the upper brain stem of cat, using a computer of average transients. One hundred evoked potentials in response to pelvic nerve stimulation are summated and averaged at each point of the brain. Evoked potentials appear in two distinct areas of the thalamus. The primary area is located in lateral region of the VPM, medial to CM. Latencies of the potential in this area are about $10 \mathrm{msec}$, the maximal amplitudes being $100 \mu \mathrm{V}$. The secondary area is located diffusely in the medial thalamic nuclei, such as NCP., CM. and also in the subthalamus. Latencies of the potential in this area are $22-26 \mathrm{msec}$, the magnitude being less than $50 \mu \mathrm{V}$. Evoked responses with latencies of about $13 \mathrm{msec}$ and low voltages are also obtained in the hypothalamus. 